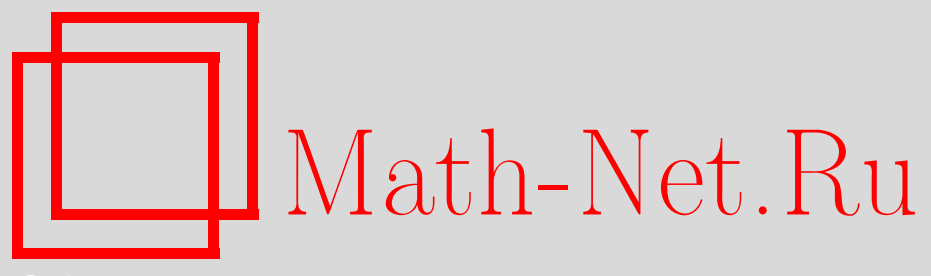

М. Л. Гандариас, М. С. Брузон, Новые решения уравнения Шварца-Кортевега-де Фриза в размерности $2+1$, полученного на основе слабых симметрий, ТМФ, 2007, том 151, номер 3, 380-390

DOI: https://doi.org/10.4213/tmf6053

Использование Общероссийского математического портала Math-Net.Ru подразумевает, что вы прочитали и согласны с пользовательским соглашением http://www . mathnet.ru/rus/agreement

Параметры загрузки:

IP: 54.80 .97 .219

26 апреля 2023 г., 06:20:23

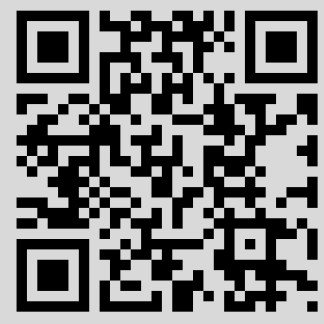




\title{
НОВЫЕ РЕШЕНИЯ УРАВНЕНИЯ ШВАРЦА-КОРТЕВЕГА-ДЕ ФРИЗА В РАЗМЕРНОСТИ $2+1$, ПОЛУЧЕННОГО НА ОСНОВЕ СЛАБЫХ СИММЕТРИЙ
}

\begin{abstract}
Рассматривается $(2+1)$-мерное интегрируемое уравнение Шварца-Кортевега-де Фриза. С помощью слабых симметрий получена система уравнений в частных производных в размерности $1+1$. Дальнейшие редукции приводят к обыкновенным дифференциальным уравнениям второго порядка, обеспечивающим построение новых решений, которые могут быть выражены через известные функции. Эти решения зависят от двух произвольных функций и одного произвольного решения волнового уравнения Римана и не могут быть получены с помощью классических и неклассических симметрий. Некоторые из найденных решений уравнения Шварца-Кортевега-де Фриза демонстрируют весьма разнообразное качественное поведение, наиболее интересными среди них являются решения вида бегущей волны и солитонные решения.
\end{abstract}

Ключевые слова: слабые симметрии, уравнения в частных производных, уединенные волны.

\section{1. ВВЕДЕНИЕ}

Хорошо известно, что уравнение Шварца-Кортевега-де Фриза (ШКдФ)

$$
\frac{\Phi_{t}}{\Phi_{x}}+\{\widehat{\Phi} ; x\}=0
$$

где

$$
\{\widehat{\Phi} ; x\}=\left(\frac{\Phi_{x x}}{\Phi_{x}}\right)_{x}-\frac{1}{2}\left(\frac{\Phi_{x x}}{\Phi_{x}}\right)^{2}
$$

- производная Шварца [1], представляет большой интерес как для физики, так и для математики. Это уравнение было введено Кричевером и Новиковым [2] и Вейссом [3] и является некоторым вариантом уравнения Кортевега-де Фриза (КдФ), который инвариантен относительно преобразования Мёбиуса, т.е. группы $P S L(2)$.

*Departamento de Matemáticas, Universidad de Cádiz, PO.BOX 40, 11510 Puerto Real, Cádiz, Spain. E-mail: marialuz.gandarias@uca.es, matematicas.casem@uca.es 
В контексте интегрируемых $(2+1)$-мерных уравнений, т.е. интегрируемых уравнений с двумя пространственными и одной временно́й переменными, Кудряшов и Пикеринг [4], а также Тода и Ю [5], используя метод Калоджеро, вывели модель

$$
W_{t}+\frac{1}{4} W_{x x z}-\frac{W_{x} W_{x z}}{2 W}-\frac{W_{x x} W_{z}}{4 W}+\frac{W_{x}^{2} W_{z}}{2 W^{2}}-\frac{W_{x}}{8}\left(\partial_{x}^{-1}\left(\frac{W_{x}^{2}}{W^{2}}\right)\right)_{z}=0,
$$

где обозначено $\partial_{x}^{-1} f=\int f d x$.

Уравнение (1) инвариантно относительно преобразования Мёбиуса и для решений вида $W(x, z, t)=\bar{W}(x+z, t)$ сводится к уравнению ШКдФ. В работе [5] была представлена соответствующая пара Лакса и было доказано, что она проходит тест Пенлеве в смысле метода Вайсса-Табора-Карневале [6].

В последние годы наблюдался постоянный интерес к вопросам, связанным с неклассическими и слабыми симметриями. Обзоры соответствующих исследований даны Олвером и Воробьевым [7] и Кларксоном [8].

Ключевым вопросом работы Олвера и Росенау [9], по-видимому, является вопрос о том, могут ли методы редукции быть унифицированы в рамках концепции дифференциальных уравнений с дополнительным условием. Олвер и Росенау [10] ввели понятие, названное ими "группами слабой симметрии". Группа слабой симметрии $G$ для системы $\Delta$ - это группа, которая не преобразует более решения в решения, а вместо этого обладает тем свойством, что $G$-инвариантные решения системы $\Delta$ находятся из редуцированной системы дифференциальных уравнений, содержащих меньшее число независимых переменных, чем исходная система $\Delta$. Обнаружилось, что неклассический метод Блумэна и Коула является частным случаем подхода, основанного на понятии групп слабой симметрии. Удивительным, по меньшей мере вначале, кажется то обстоятельство, что любое решение системы $\Delta$ может быть получено как решение, инвариантное относительно некоторой группы слабой симметрии, и что более или менее все группы являются группами слабой симметрии.

Воробьев [11] провел исследование неклассических инфинитезимальных слабых симметрий, введенных Олвером и Росенау, а также неполных симметрий, введенных им самим. Для семейства нелинейных уравнений теплопроводности вида $u_{t}=$ $\left(k(u) u_{x}\right)_{x}+q(u)$ он указал такие пары функций $(k(u), q(u))$, что соответствующие уравнения допускают нетривиальные двумерные модули неполных симметрий, которые приводят к явным решениям.

Джамай и Воробьев [12] проанализировали неклассические инфинитезимальные слабые симметрии дифференциальных уравнений в частных производных (ДУЧП), предложенные Олвером и Росенау. Для случая ДУЧП с двумя независимыми переменными они показали, что получение таких симметрий эквивалентно получению двумерных модулей неклассических неполных симметрий.

Центральным вопросом работы [13] является вопрос о том, какие дополнительные условия допустимы и позволяют получить классические решения для данных дифференциальных уравнений. Работа показала, что слабые симметрии представляют не только академический интерес, но необходимы для нахождения всех решений уравнений Навье-Стокса, получаемых методом частичного обращения.

В работе [14] представлен обзор различных обобщений классических симметрий Ли: условные симметрии, неполные симметрии и $\lambda$-симметрии вместе с соответствующими критериями и процедурами. Были рассмотрены “слабые" и "сильные" 
классические и условные симметрии и предложены неполные и слабые условные $\lambda$ симметрии. Автор также рассмотрел связи между $\lambda$-симметриями и условными и классическими симметриями. Были приведены простые примеры симметрий каждого типа. В частности, был дан пример симметрии, которая может рассматриваться и как слабая условная симметрия, и как неполная симметрия для одного и того же уравнения, при этом разным типам симметрии отвечают различные множества решений.

С помощью преобразований

$$
W=\phi_{x}, \quad \phi=e^{\psi}, \quad \psi_{x}=u, \quad \psi_{t}=v
$$

уравнение (1) может быть представлено в виде системы

$$
\begin{aligned}
4 u^{2} v_{x}-4 u u_{x} v+u^{2} u_{x x z}-u u_{x x} u_{z}-3 u u_{x} u_{x z}+3 u_{x}^{2} u_{z}-u^{4} u_{z} & =0 \\
u_{t}-v_{x} & =0 .
\end{aligned}
$$

Эта система была исследована в работе [15] с помощью классического метода групп Ли инфинитезимальных преобразований, а в работе [16] - с использованием неклассического метода.

Уравнение (1) возникает в нелокальной форме, однако может также быть записано в следующем локальном виде:

$$
\begin{aligned}
\Delta \equiv 8 & \frac{u_{t x}}{u_{x}}-8 \frac{u_{t} u_{x x}}{u_{x}^{2}}+2 \frac{u_{x x x z}}{u_{x}}-2 \frac{u_{x x} u_{x x z}}{u_{x}^{2}}-4 \frac{u_{x x z}}{u}+6 \frac{u_{x} u_{x z}}{u^{2}}- \\
& -2 \frac{u_{z} u_{x x x}}{u u_{x}}-2 \frac{u_{x x} u_{x z}}{u u_{x}}+2 \frac{u_{z} u_{x x}^{2}}{u u_{x}^{2}}+6 \frac{u_{z} u_{x x}}{u^{2}}-6 \frac{u_{z} u_{x}^{2}}{u^{3}}=0 .
\end{aligned}
$$

В настоящей работе мы сконцентрируем свое внимание на получении таких решений уравнения (1), которые не могут быть получены ни с помощью классического метода групп Ли преобразований [15], ни с помощью неклассического метода Бламэна и Коула [17]. Мы используем метод слабых симметрий, предложенный Олвером и Росенау [9], [10]. Такой подход заключается в вычислении симметрий уравнения (4), дополненного некоторыми дифференциальными ограничениями, выбранными так, чтобы ослабить критерий инвариантности для основной системы и обеспечить существование более широких точечных групп Ли симметрий для расширенной системы.

\section{2. СЛАБЫЕ СИММЕТРИИ УРАВНЕНИЯ ШКДФ В РАЗМЕРНОСТИ $2+1$}

Чтобы построить новые решения уравнения (1), получим слабые симметрии уравнения ШКдФ (4). Порядок действий следующий. Добавим к ДУЧП некоторое дополнительное условие. Потребовав, чтобы как уравнение (4), так и дополнительное условие были инвариантны относительно однопараметрической группы Ли инфинитезимальных преобразований в пространстве переменных $(x, t, z, u)$, заданных формулами

$$
\begin{aligned}
x^{*} & =x+\varepsilon \xi(x, z, t, u)+\mathcal{O}\left(\varepsilon^{2}\right), \\
z^{*} & =z+\varepsilon \eta(x, z, t, u)+\mathcal{O}\left(\varepsilon^{2}\right), \\
t^{*} & =t+\varepsilon \tau(x, z, t, u)+\mathcal{O}\left(\varepsilon^{2}\right), \\
u^{*} & =u+\varepsilon \phi(x, z, t, u)+\mathcal{O}\left(\varepsilon^{2}\right),
\end{aligned}
$$


где $\varepsilon$ - параметр группы, получаем переопределенную нелинейную систему уравнений для инфинитезимальных элементов $\xi(x, z, t, u), \quad \eta(x, z, t, u), \quad \tau(x, z, t, u)$ и $\phi(x, z, t, u)$.

Для применения метода на практике мы использовали пакет программ MACSYMA symmgrp.max [18].

Мы реализуем процедуру с двумя различными типами дополнительных условий. В первом случае мы рассматриваем дополнительное условие первого порядка, а во втором - дополнительное условие второго порядка.

Редукция 1. Применение классического метода к уравнению (4) и дополнительному условию

$$
\alpha(x, z, t, u) \frac{\partial u}{\partial x}+\beta(x, z, t, u) \frac{\partial u}{\partial z}+\frac{\partial u}{\partial t}-\Psi(x, z, t, u)=0
$$

дает систему из 23 уравнений для инфинитезимальных элементов $\xi(x, z, t, u)$, $\eta(x, z, t, u), \tau(x, z, t, u)$ и $\phi(x, z, t, u)$ и функций $\alpha(x, z, t, u), \beta(x, z, t, u)$ и $\Psi(x, z, t, u)$. Из этой системы получаем, что $\xi=\gamma(t) x+\zeta(t), \eta=\eta(t, z), \tau=\tau(t), \phi=r(z, t) u$ и что функции $\gamma, \eta, \tau, \zeta, r, \alpha, \beta$ и $\Psi$ удовлетворяют следующим уравнениям:

$$
\begin{aligned}
& \beta_{x} \gamma x+\beta_{u} r u+\beta_{x} \zeta+2 \beta \gamma+\beta_{t} \tau+\beta_{z} \eta=0, \\
& \beta_{x x} \gamma x+\beta_{u x} r u+\beta_{x x} \zeta+3 \beta_{x} \gamma+\beta_{t x} \tau+\beta_{z x} \eta=0, \\
& \beta_{u x} \gamma x+\beta_{u u} r u+\beta_{u} r+\beta_{u x} \zeta+2 \beta_{u} \gamma+\beta_{t u} \tau+\beta_{u z} \eta=0, \\
& \alpha_{u x} \gamma x+\alpha_{u u} r u+\alpha_{u} r+\alpha_{u x} \zeta+\alpha_{u} \gamma+\alpha_{t u} \tau+\alpha_{u} \eta_{z}+\alpha_{u z} \eta=0, \\
& \gamma \Psi_{x} x-\beta r_{z} u+\Psi_{u} r u-\psi r+\eta \Psi_{z}+\zeta \Psi_{x}+\tau \Psi_{t}+2 \gamma \psi+\eta_{z} \psi=0, \\
& \gamma \Psi_{x x} x-\beta_{x} r_{z} u+\Psi_{u x} r u-\Psi_{x} r+\zeta \Psi_{x x}+\eta \Psi_{x z}+3 \gamma \Psi_{x}+\eta_{z} \Psi_{x}+\tau \Psi_{t x}=0, \\
& \Psi_{u x} x \gamma-\alpha_{x x} x \gamma+2 \Psi_{u} \gamma-2 \alpha_{x} \gamma+\Psi_{u x} \zeta-\alpha_{x x} \zeta+r \Psi_{u u} u-\beta_{u} r_{z} u-\alpha_{u x} r u+ \\
& \quad+\eta \Psi_{u z}+\eta_{z} \Psi_{u}+\tau \Psi_{t u}-\beta r_{z}-\alpha_{t x} \tau-\alpha_{x} \eta_{z}-\alpha_{x z} \eta=0 .
\end{aligned}
$$

Решение этой системы в общем случае очень сложно. Однако если $\alpha=\alpha(t)$ и $\psi=0$, то одно решение характеристических уравнений есть

$$
\xi=\zeta(t), \quad \eta=\eta(z, t), \quad \tau=1, \quad \phi=0,
$$

где $\zeta$ и $\eta$ - произвольные функции, а функция $\beta(z, t)$ должна удовлетворять уравнению $\beta_{t}+\eta \beta_{z}=0$.

Равенства (7) позволяют легко проверить, что эти инфинитезимальные элементы не удовлетворяют неклассическим определяющим уравнениям. Разрешая соответствующее поверхностное условие, получаем редукции симметрии

$$
w_{1}=x-\int \zeta(t) d t, \quad w_{2}=w_{2}(t, z), \quad u=f\left(w_{1}, w_{2}\right),
$$

где функция $\eta$ удовлетворяет уравнению $w_{2, t}+\eta w_{2, z}=0$, а функция $f$ удовлетворяет следующей $(1+1)$-мерной системе ДУЧП:

$$
\begin{gathered}
-f^{2} f_{w_{2}} f_{w_{1}} f_{w_{1} w_{1} w_{1}}+f^{2} f_{w_{2}} f_{w_{1} w_{1}}^{2}+3 f f_{w_{2}} f_{w_{1}}^{2} f_{w_{1} w_{1}}-f^{2} f_{w_{2} w_{1}} f_{w_{1}} f_{w_{1} w_{1}}- \\
-f^{3} f_{w_{1} w_{1}} f_{w_{1} w_{1}}-3 f_{w_{2}} f_{w_{1}}^{4}+3 f f_{w_{2} w_{1}} f_{w_{1}}^{3}-2 f^{2} f_{w_{2} w_{1} w_{1}} f_{w_{1}}^{2}+ \\
+f^{3} f_{w_{2} w_{1} w_{1} w_{1}} f_{w_{1}}=0 \\
4 f^{3} f_{w_{2} w_{1}} f_{w_{1}}-4 f^{3} f_{w_{2}} f_{w_{1} w_{1}}=0 .
\end{gathered}
$$


Редукция 1.1. Редуцированная система ДУЧП (9) в пространстве $1+1$ переменных инвариантна относительно трансляций и допускает дальнейшие редукции к системе обыкновенных дифференциальных уравнений (ОДУ). В результате получаем переменные

$$
\vartheta=w_{1}+w_{2}, \quad f=g(\vartheta)
$$

и автономное ОДУ

$$
-g^{3} g^{\prime} g^{\prime \prime \prime \prime}+g^{3} g^{\prime \prime} g^{\prime \prime \prime}+3 g^{2}\left(g^{\prime}\right)^{2} g^{\prime \prime \prime}-6 g\left(g^{\prime}\right)^{3} g^{\prime \prime}+3\left(g^{\prime}\right)^{5}=0 .
$$

Делением на $g^{2}\left(g^{\prime}\right)^{2}$, однократным интегрированием по переменной $\vartheta$ и последующим умножением на $g^{-2} g^{\prime}$ уравнение (11) может быть сведено к ОДУ второго порядка

$$
g^{\prime \prime}=\frac{3}{2} \frac{\left(g^{\prime}\right)^{2}}{g}-k_{2} g+k_{1},
$$

где $k_{1}$ и $k_{2}$ - произвольные постоянные.

Умножая уравнение (12) на $g^{-3} g^{\prime}$ и интегрируя один раз по переменной $\vartheta$, получаем

$$
\left(g^{\prime}\right)^{2}=-2 k_{2} g^{2}+k_{1} g+2 k_{3} g^{3} .
$$

Интегрирование может быть доведено до конца в эллиптических функциях.

Редукция 1.2. Редуцированная система ДУЧП (9) в пространстве $1+1$ переменных инвариантна относительно скейлинговой группы и допускает дальнейшие редукции к системе ОДУ. Имеем

$$
\vartheta=w_{2}^{n} w_{1}, \quad f=w_{2}^{m} g
$$

и систему ОДУ

$$
\begin{gathered}
g^{3} g^{\prime} g^{\prime \prime \prime \prime} \vartheta-g^{3} g^{\prime \prime} g^{\prime \prime \prime} \vartheta-3 g^{2}\left(g^{\prime}\right)^{2} g^{\prime \prime \prime} \vartheta+6 g\left(g^{\prime}\right)^{3} g^{\prime \prime} \vartheta-3\left(g^{\prime}\right)^{5} \vartheta+3 g^{3} g^{\prime} g^{\prime \prime \prime}- \\
-2 g^{3}\left(g^{\prime \prime}\right)^{2}-5 g^{2}\left(g^{\prime}\right)^{2} g^{\prime \prime}+3 g\left(g^{\prime}\right)^{4}=0 \\
m g g^{\prime \prime}-(m+n)\left(g^{\prime}\right)^{2}=0 .
\end{gathered}
$$

Редукция 2. Применение классического метода к уравнению (4) и дополнительным условиям

$$
\beta \frac{\partial u}{\partial z}+\frac{\partial u}{\partial t}=0, \quad \frac{\partial u}{\partial x \partial z}=0
$$

дает систему из 10 уравнений для инфинитезимальных элементов $\xi(x, z, t, u)$, $\eta(x, z, t, u), \tau(x, z, t, u)$ и $\phi(x, z, t, u)$ и функции $\beta(x, z, t, u)$. Из этой системы получаем, что

$$
\xi=\gamma(z, t) x+\zeta(z, t), \quad \eta=\eta(z, t), \quad \tau=\tau(x, z, t, u), \quad \phi=\rho(z, t) u^{3}+\delta(z, t) u,
$$

где $\beta, \gamma, \tau, \zeta, \rho, \delta$ и $\eta$ суть функции, которые должны удовлетворять следующим уравнениям:

$$
\begin{aligned}
-\beta_{x} u x \gamma-2 \beta u \gamma-\beta_{x} u \zeta-\beta_{u} \rho u^{4}+2 \beta \rho u^{3}-\beta_{u} \delta u^{2}-\beta_{t} \tau u-\beta_{z} \eta u=0, & \\
\beta_{u x} u x \gamma+ & 2 \beta_{u} u \gamma+\beta_{u x} u \zeta+\beta_{u u} \rho u^{4}+\beta_{u} \rho u^{3}+6 \beta \rho u^{2}+\beta_{u u} \delta u^{2}+\beta_{t u} \tau u+ \\
& +\beta_{u z} \eta u+\beta_{u} \delta u=0, \\
-\beta_{x x} u x \gamma- & 3 \beta_{x} u \gamma-\beta_{x x} u \zeta-\beta_{u x} \rho u^{4}+2 \beta_{x} \rho u^{3}-\beta_{u x} \delta u^{2}-\beta_{t x} \tau u-\beta_{x z} \eta u=0 .
\end{aligned}
$$


Мы не можем решить систему (16) в общем случае по причине ее сложности. Однако если $\beta=\beta(z, t)$, то решение имеет вид

$$
\xi=\zeta(z, t), \quad \eta=\eta(z, t), \quad \tau=1, \quad \phi=\delta(z) u .
$$

Разрешая соответствующее поверхностное условие, получаем

$$
w_{1}=x+b(z, t), \quad w_{2}=a(t, z), \quad u=\rho(z) f\left(w_{1}, w_{2}\right),
$$

что приводит к следующей системе ДУЧП двух независимых переменных, в которой $a_{t}-k_{1} a^{2} a_{z}=0$, а $\rho=\rho(z)$ и $b=b(z, t)$ - произвольные функции:

$$
\begin{gathered}
f^{3} f_{w_{1}} f_{w_{1} w_{1} w_{1} w_{1}}-f^{3} f_{w_{1} w_{1}} f_{w_{1} w_{1} w_{1}}-3 f^{2} f_{w_{1}}^{2} f_{w_{1} w_{1} w_{1}}+6 f f_{w_{1}}^{3} f_{w_{1} w_{1}}-3 f_{w_{1}}^{5}=0, \\
-4 k_{1} f^{3} f_{w_{1} w_{1}} f_{w_{2}} w_{2}^{2}+4 k_{1} f^{3} f_{w_{1}} f_{w_{1} w_{2}} w_{2}^{2}-f^{2} f_{w_{1}} f_{w_{1} w_{1} w_{1}} f_{w_{2}}+f^{2} f_{w_{1} w_{1}}^{2} f_{w_{2}}+ \\
+3 f f_{w_{1}}^{2} f_{w_{1} w_{1}} f_{w_{2}}-3 f_{w_{1}}^{4} f_{w_{2}}+f^{3} f_{w_{1}} f_{w_{1} w_{1} w_{1} w_{2}}-f^{3} f_{w_{1} w_{1}} f_{w_{1} w_{1} w_{2}}- \\
-2 f^{2} f_{w_{1}}^{2} f_{w_{1} w_{1} w_{2}}-f^{2} f_{w_{1}} f_{w_{1} w_{2}} f_{w_{1} w_{1}}+3 f f_{w_{1}}^{3} f_{w_{1} w_{2}}=0 .
\end{gathered}
$$

Редукция 2.1. Система (17) инвариантна относительно скейлинговой группы. Следовательно, решая характеристическое уравнение, мы получаем редукцию симметрии

$$
\vartheta=w_{1} w_{2}, \quad f=h(\vartheta),
$$

что дает следующую систему ОДУ:

$$
\begin{aligned}
h^{3} h^{\prime} h^{\prime \prime \prime \prime}-h^{3} h^{\prime \prime} h^{\prime \prime \prime}-3 h^{2} h^{\prime 2} h^{\prime \prime \prime}+6 h h^{3} h^{\prime \prime}-3 h^{5} & =0, \\
3 h^{2} h^{\prime} h^{\prime \prime \prime}-2 h^{2} h^{\prime \prime 2}-5 h h^{2} h^{\prime \prime}+3 h^{4}+4 k_{1} h^{2} h^{2} & =0 .
\end{aligned}
$$

Проинтегрировав первое уравнение один раз по переменной $\vartheta$ и сделав замену переменной $h^{\prime} / h=g$, получаем систему ОДУ второго порядка:

$$
\begin{aligned}
g g^{\prime \prime}-g^{2}-\frac{g^{4}}{4}+k_{2} & =0, \\
3 g g^{\prime \prime}-2 g^{\prime 2}-g^{4}+4 k_{1} g^{2} & =0 .
\end{aligned}
$$

Редукция 2.2. Система (17) инвариантна относительно скейлинговой группы. Следовательно, решая характеристическое уравнение, получаем редукцию симметрии

$$
\vartheta=w_{1} w_{2}, \quad f=\frac{1}{w_{2}} h(\vartheta),
$$

что дает следующую систему ОДУ:

$$
\begin{aligned}
h^{3} h^{\prime} h^{\prime \prime \prime \prime}-h^{3} h^{\prime \prime} h^{\prime \prime \prime}-3 h^{2}\left(h^{\prime}\right)^{2} h^{\prime \prime \prime}+6 h\left(h^{\prime}\right)^{3} h^{\prime \prime}-3\left(h^{\prime}\right)^{5} & =0, \\
3 h^{2} h^{\prime} h^{\prime \prime \prime}-2 h^{2}\left(h^{\prime \prime}\right)^{2}+4 k_{1} h^{3} h^{\prime \prime}-5 h\left(h^{\prime}\right)^{2} h^{\prime \prime}+3\left(h^{\prime}\right)^{4} & =0 .
\end{aligned}
$$

Сделав замену переменной $h_{\vartheta} / h=g$ и проинтегрировав один раз по переменной $\vartheta$, получаем систему

$$
\begin{array}{r}
g g^{\prime \prime}-g^{\prime 2}-\frac{g^{4}}{4}+k_{2}=0, \\
3 g g^{\prime \prime}-2 g^{2}-4 k_{1} n g^{\prime}-g^{4}+4 k_{1} g^{2}=0 .
\end{array}
$$




\section{3. НЕКОТОРЫЕ ТОЧНЫЕ РЕШЕНИЯ}

Представим далее некоторые точные решения ОДУ второго порядка, а также соответствующие решения уравнения ШКдФ в размерности $2+1$.

Редукция 1.1. Уравнение (11) может быть проинтегрировано в эллиптических функциях:

$$
g_{1}=\operatorname{sn}^{ \pm 2}(\vartheta \mid m), \quad g_{2}=\operatorname{cn}^{ \pm 2}(\vartheta \mid m), \quad g_{3}=\operatorname{dn}^{ \pm 2}(\vartheta \mid m)
$$

Ясно, что любое вырождение (рациональное, гиперболическое или тригонометрическое) эллиптических функций также дает решение уравнения (11):

$$
\begin{gathered}
g=\frac{k_{1}}{k_{2}} \operatorname{th}^{ \pm 2}\left(\frac{\sqrt{-k_{2}} \vartheta}{2}\right), \quad g=-\frac{k_{1}}{k_{2}} \operatorname{tg}^{ \pm 2}\left(\frac{\sqrt{k_{2}} \vartheta}{2}\right), \quad g=-\frac{k_{1}}{2 k_{2}} \operatorname{sh}^{ \pm 2}\left(\frac{\sqrt{k_{2}} \vartheta}{\sqrt{2}}\right), \\
g=\frac{k_{1}}{2 k_{2}} \operatorname{ch}^{ \pm 2}\left(\frac{\sqrt{k_{2}} \vartheta}{\sqrt{2}}\right), \quad g=\frac{k_{1}}{2 k_{2}} \sin ^{ \pm 2}\left(\frac{\sqrt{-k_{2}} \vartheta}{\sqrt{2}}\right), \quad g=\frac{k_{1}}{2 k_{2}} \cos ^{ \pm 2}\left(\frac{\sqrt{-k_{2}} \vartheta}{\sqrt{2}}\right), \\
g=k_{3} \vartheta^{ \pm 2}, \quad g=k_{4} e^{ \pm \sqrt{2 k_{2}} \vartheta} .
\end{gathered}
$$

Рассмотрев соответствующие редукции симметрии (8) и (10), получаем, что точные решения уравнения (1) могут быть записаны в виде

$$
\begin{gathered}
u=\mathrm{sn}^{ \pm 2}(x+a(z, t)+\delta(t) \mid m), \quad u=\operatorname{dn}^{ \pm 2}(x+a(z, t)+\delta(t) \mid m), \\
u=\operatorname{sn}^{ \pm 2}(x+a(z, t)+\delta(t) \mid m),
\end{gathered}
$$

где $a(z, t)=w_{2}(z, t)$ и $\delta(t)=-\int \zeta(t) d t$,

$$
\begin{aligned}
& u=\frac{k_{1} \rho(z)}{k_{2}} \operatorname{th}^{ \pm 2}\left(\frac{\sqrt{-k_{2}}(x+a(z, t)+\delta(t))}{2}\right), \\
& u=-\frac{k_{1} \rho(z)}{k_{2}} \operatorname{tg}^{ \pm 2}\left(\frac{\sqrt{k_{2}}(x+a(z, t)+\delta(t))}{2}\right), \\
& u=k_{3} \rho(z) \operatorname{sch}^{ \pm 2}\left(\frac{\sqrt{k_{2}}(x+a(z, t)+\delta(t))}{\sqrt{2}}\right), \\
& u=k_{3} \rho(z) \operatorname{csch}^{ \pm 2}\left(\frac{\sqrt{k_{2}}(x+a(z, t)+\delta(t))}{\sqrt{2}}\right), \\
& u=k_{3} \rho(z) \sec ^{ \pm 2}\left(\frac{\sqrt{-k_{2}}(x+a(z, t)+\delta(t))}{\sqrt{2}}\right), \\
& u=k_{3} \rho(z) \operatorname{cosec}^{ \pm 2}\left(\frac{\sqrt{-k_{2}}(x+a(z, t)+\delta(t))}{\sqrt{2}}\right), \\
& u=k_{3} \rho(z)(x+a(z, t)+\delta(t))^{ \pm 2}, \\
& u=\rho(z) e^{\sqrt{-2 k_{1}}(x+a(z, t)+\delta(t))} .
\end{aligned}
$$


Редукция 1.2. Совместность уравнений системы (15) предполагает выполнение условия $m= \pm 2 n$, следовательно, ее решения суть

$$
g=k_{1}\left(\vartheta+k_{2}\right)^{ \pm 2}
$$

а решения уравнения (1) суть

$$
u=k_{1}\left(\frac{a^{n}(x-\delta)+k_{2}}{a^{n}}\right)^{ \pm 2} .
$$

Редукция 2.1. Имеем следующие решения системы (18):

1) если $k_{2}=0$, то $g=4 \sqrt{k_{1}} \operatorname{cosec}\left(2 \sqrt{k_{1}} \vartheta\right), g=4 \sqrt{-k_{1}} \operatorname{csch}\left(2 \sqrt{-k_{1}} \vartheta\right)$;

2) если $k_{2}=4 k_{1}^{2}$ и $k_{1}=k^{2} / 4$, то $g=k$.

Из условия $h^{\prime} / h=g$ получаем соответственно

$$
h=c \operatorname{tg}^{ \pm 2}\left(\sqrt{k_{1}} \vartheta\right), \quad h=c \operatorname{th}^{ \pm 2}\left(\sqrt{-k_{1}} \vartheta\right), \quad h=c e^{ \pm k \vartheta} .
$$

В результате имеем следующие решения уравнения (1):

$$
\begin{gathered}
u=\rho(z) \operatorname{tg}^{ \pm 2}\left(a(z, t) \sqrt{k_{1}}(x+b(z, t))\right), \quad u=\rho(z) \operatorname{th}^{ \pm 2}\left(a(z, t) \sqrt{-k_{1}}(x+b(z, t))\right), \\
u=e^{ \pm 2 \sqrt{k_{1}} a(z, t)(x+b(z, t))}
\end{gathered}
$$

где функция $a=w_{2}$ и удовлетворяет волновому уравнению Римана $a_{t}-k_{1} a^{2} a_{z}=0$, а $\rho=\rho(z)$ и $b=b(z, t)$ - произвольные функции.

Мы видим, что функция $b=b(z, t)$ определяет смещение волны, функция $\rho=\rho(z)$ модулирует амплитуду волны, а функция $a=a(z, t)$ - ее скорость.

Редукция 2.2. Имеем следующие решения системы (19):

$$
h=\sin \left(2 \sqrt{-k_{1}} \vartheta\right) \pm 1, \quad h=\cos ^{ \pm 2}\left(\sqrt{-k_{1}} \vartheta\right), \quad h=\operatorname{ch}^{ \pm 2}\left(\sqrt{k_{1}} \vartheta\right) .
$$

Решения уравнения (1) имеют вид соответственно

$$
\begin{aligned}
& u=\frac{\rho(z) \sin \left(2 \sqrt{-k_{1}} a(z, t)(x+b(z, t)) \pm 1\right)}{a(z, t)}, \\
& u=\frac{\rho(z) \cos ^{ \pm 2}\left(\sqrt{-k_{1}} a(z, t)(x+b(z, t))\right)}{a(z, t)}, \\
& u=\frac{\rho(z) \operatorname{ch}^{ \pm 2}\left(\sqrt{k_{1}} a(z, t)(x+b(z, t))\right)}{a(z, t)},
\end{aligned}
$$

где функция $a=w_{2}$ и является произвольным решением волнового уравнения Римана $a_{t}-k_{1} a^{2} a_{z}=0$.

Мы видим, что функция $b=b(z, t)$ определяет смещение волны, а функция $a=$ $a(z, t)$ модулирует амплитуду и скорость волны.

На рис. 1 представлено решение

$$
u=\operatorname{sch}^{2}\left(x+\sin \left(\frac{z^{2} t^{2}}{4}\right)\right)
$$




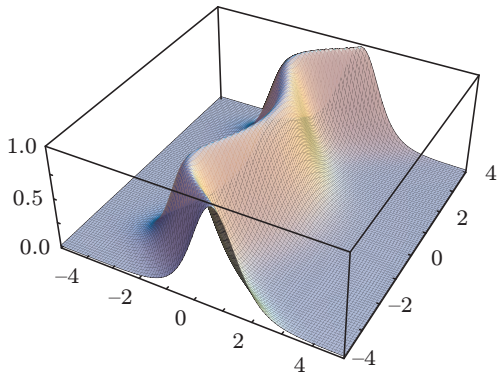

$a$

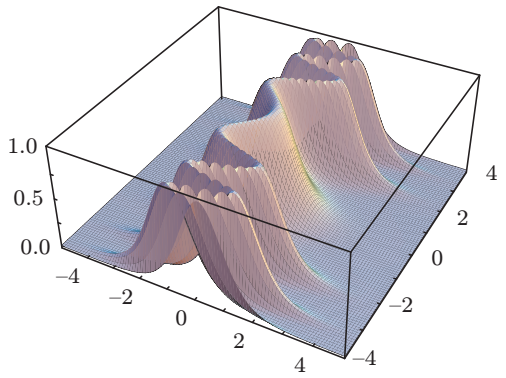

6

Рис. 1. Решение (24) в моменты времени $t=1$ (а) и $t=2$ (б).

для моментов времени $t=1$ и $t=2$, соответственно. Видно, что это решение эволюционирует без ограничений на $x=b(z, t)$; решение такого рода не может быть получено ни классическим методом Ли, ни с помощью неклассического метода.

На рис. 2 представлено решение

$$
u=\operatorname{sch}^{2}(z) \operatorname{th}^{2}\left(x+\sin ^{2}\left(\frac{z^{2} t^{2}}{4}\right)\right)
$$

для момента времени $t=1$ и соответствующие профили сечения $x=0$ в моменты времени $t=1$ и $t=2$, отражающие эволюцию во времени.

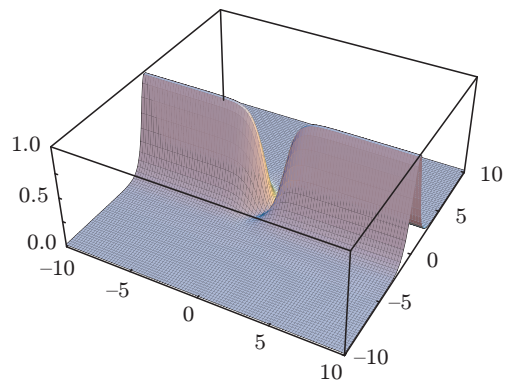

$a$

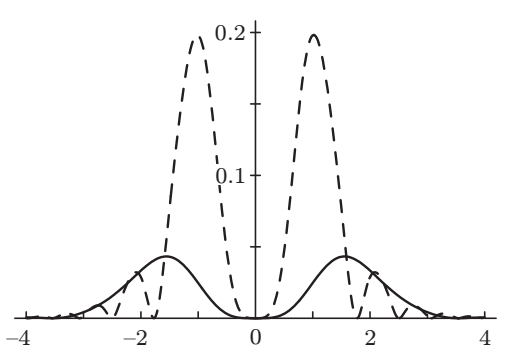

б

Рис. 2. Решение (25) в момент времени $t=1$ (а) и соответствующие профили сечения $x=0$ в моменты времени $t=1$ (сплошная кривая) и $t=2$ (штриховая кривая) (б).

На рис. 3 представлено решение

$$
u=\rho(z) \operatorname{sch}^{2}(x+0.2 \operatorname{th} z+0.2 \operatorname{th}(z+6)-0.1 \operatorname{th}(z-8)+\sin t),
$$

где $\rho(z)=0.2 \mathrm{sch}^{2} z+0.2 \mathrm{sch}^{2}(z+6)-0.1 \mathrm{sch}^{2}(z-8)$ для момента времени $t=-2$ и соответствующие профили сечения $x=0$ в моменты времени $t=-2$ и $t=2$, которые отражают эволюцию во времени.

На рис. 4 представлено решения Якоби

$$
u=\operatorname{sn}^{2}\left(\begin{array}{l|l}
x+z^{2} t & \frac{1}{3}
\end{array}\right)
$$

для моментов времени $t=0.5$ и $t=2$. 


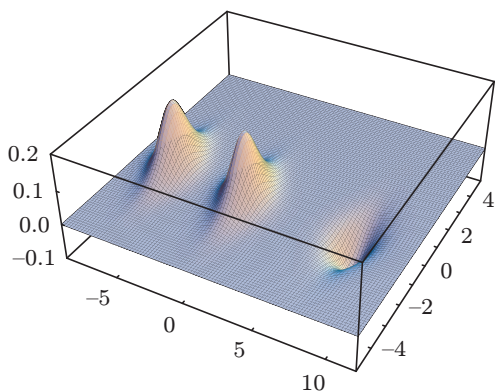

$a$

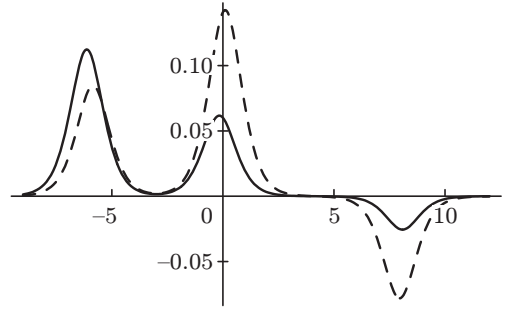

б

Рис. 3. Решение (26): три дромиона в момент времени $t=-2$ (а) и соответствующие профили сечения $x=0$ в моменты времени $t=-2$ (штриховая кривая) и $t=2$ (сплошная кривая) (б).

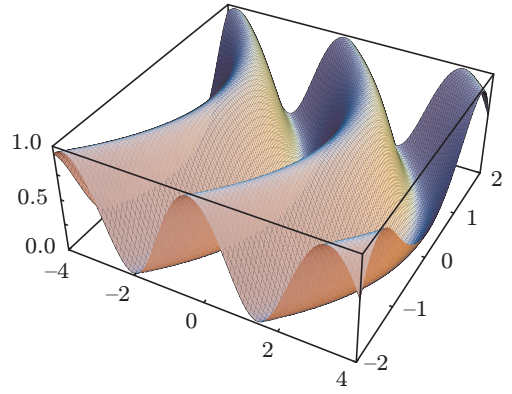

$a$

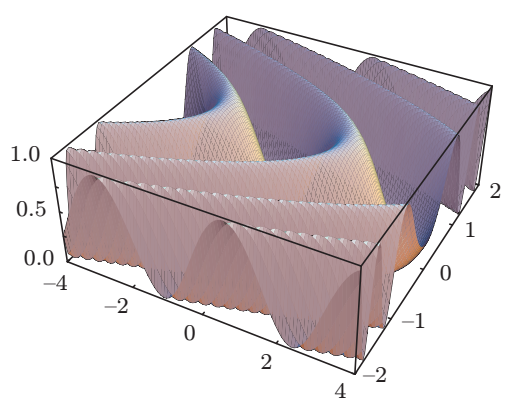

б

Рис. 4. Решение Якоби (27) для моментов времени $t=0.5$ (а) и $t=2$ (б).

\section{4. ЗАКЛЮЧЕНИЕ}

В данной работе было рассмотрено $(2+1)$-мерное интегрируемое уравнение ШКдФ. С помощью слабых симметрий и последующих редукций были выведены ОДУ второго порядка, которые позволили получить новые решения, выражающиеся через известные функции.

Соответствующие решения уравнения (1) зависят от двух произвольных функций $\rho=\rho(z), b=b(z, t)$ и от произвольного решения $a=a(z, t)$ волнового уравнения Римана $a_{t}-k_{1} a^{2} a_{z}=0$. Таким образом, некоторые из найденных решений уравнения ШКдФ демонстрируют весьма разнообразное качественное поведение: наиболее интересными среди них являются солитонные и дромионные решения. Мы видели, что произвольная функция $b=b(z, t)$ определяет для этих решений смещение волны, а произвольное решение волнового уравнения Римана модулирует амплитуду волны и ее скорость. Данные решения уравнения (1) не могут быть получены ни классическим методом групп Ли преобразований [15], ни с помощью неклассического метода Бламэна и Коула [16]. 


\section{Список литературы}

[1] E. Hille, Analytic Function Theory, v. II, Ginn, Boston-New York-Chicago, 1962; H. Schwerdtfeger, Geometry of Complex Numbers. Circle Geometry, Moebius Transformation, nonEuclidean Geometry, Dover, New York, 1979.

[2] И. М. Кричевер, С. П. Новиков, УМН, 35:6 (1980), 47.

[3] J. Weiss, J. Math. Phys., 24 (1983), 1405.

[4] N. Kudryashov, P. Pickering, J. Phys. A, 31 (1998), 9505.

[5] K. Toda, S. Yu, J. Math. Phys., 41 (2000), 4747.

[6] J. Weiss, J. M. Tabor, G. Carnevale, J. Math. Phys., 24 (1983), 522.

[7] P. J. Olver, E. M. Vorob'ev, "Nonclassical and conditional symmetries", CRC Handbook of Lie Group Analysis of Differential Equations, v. 3, ed. N. H. Ibragimov, CRC, Boca Raton, FL, 1996, 291.

[8] P. A. Clarkson, Chaos Solitons Fractals, 5 (1995), 2261.

[9] P. J. Olver, P. Rosenau, Phys. Lett. A, 144 (1986), 107.

[10] P. J. Olver, P. Rosenau, SIAM J. Appl. Math., 47 (1987), 263.

[11] E. M. Vorob'ev, J. Nonlinear Math. Phys., 3 (1996), 330.

[12] A. V. Dzhamay, E. M. Vorob'ev, J. Phys. A, 27 (1994), 5541.

[13] G. Saccomandi, Note Mat., 23:2 (2004), 217.

[14] G. Cicogna, Note Mat., 23:2 (2005), 15.

[15] J. Ramirez, M. S. Bruzon, C. Muriel, M. L. Gandarias, J. Phys. A, 36 (2003), 1467.

[16] J. Ramírez, J.L. Romero, M.S. Bruzón, M.L. Gandarias, Chaos Solitons Fractals, 32 (2007), 682 .

[17] G. W. Bluman, J. D. Cole, J. Math. Mech., 18 (1969), 1025.

[18] B. Champagne, W. Hereman, P. Winternitz, Comput. Phys. Comm., 66 (1991), 319. 